\title{
A Novel Subband Fractional Delay Algorithm Based on the Filterbank of Cochlear Implant
}

\section{Yousheng Chen}

Shenzhen Institute of Information Technology https://orcid.org/0000-0001-8658-6521

Yan Chen ( $\nabla$ chenyan@sziit.edu.cn )

Shenzhen Institute of Information Technology https://orcid.org/0000-0002-1959-1889

\section{Research}

Keywords: cochlear implant, subband fractional delay, delay parameter, error minimization

Posted Date: April 10th, 2020

DOI: https://doi.org/10.21203/rs.3.rs-21519/v1

License: (c) (i) This work is licensed under a Creative Commons Attribution 4.0 International License. Read Full License 


\title{
A Novel Subband Fractional Delay Algorithm Based on the Filterbank of Cochlear Implant
}

\author{
Yousheng Chen ${ }^{1}$, Yan Chen ${ }^{2 *}$
}

*Correspondence: Yan Chen
chenyan@sziit.edu.cn
Shenzhen Institute of Information
Technology, Shenzhen 518000 ,
China
Full list of author information is
available at the end of the article.

\begin{abstract}
Background: In recent years, microphone array method is gradually applied to speech enhancement of cochlear implant, and the delay parameter is the main parameter of microphone array beamforming technology. Due to the size limitation of cochlear implant, the microphone spacing is very small. In algorithm implementation, delay parameter usually corresponds to fractional sampling point. It is necessary to use fractional delay filter to realize the interpolation of integer sampling points. The traditional fractional delay method is to interpolate sampling points in the whole frequency band of speech. However, the speech frequency band itself is very wide, so the error of the present fractional delay method in cochlear is still large.
\end{abstract}

Methods: We propose a fractional delay algorithm based on the filter bank of cochlear implant. The algorithm deduces and calculates the mathematical expression of the fractional delay filter of each subband, and forms a full band fractional delay filter algorithm to minimize the delay error of the whole band.

Realization and results: Through the analysis of the system response curve and the calculation of the delay error, it can be seen that the system response corresponding to the fractional delay of each subband in the cochlear filter bank has only a small deviation from the ideal fractional delay filter. Therefore, the error of the fractional delay filter designed in this paper is very small, which can meet the requirements of cochlear implant using microphone array technology for the precision of delay parameters.

Discussions: In this paper, the implementation algorithm of subband fractional delay filter is applied to signal acquisition of cochlear implant. Considering the space condition and delay parameters in the actual application scenario, the value of fractional delay can be any continuous real number between 0 and 3 , and the error situation of the algorithm can be calculated and analyzed in this range. If the algorithm is extended to other applications, the numerical range of fractional delay can be extended. From the statistics of the average error, it can be seen that the average error of the proposed algorithm in the whole frequency band is extremely small, which can meet the needs of the accuracy of the delay parameters in the application of cochlear implant.

Conclusions: The proposed fractional delay filter based on the minimum subband error of cochlear implant can not only realize the local fractional delay minimization, but also the error minimization of the whole frequency band.

Keywords: cochlear implant; subband fractional delay; delay parameter; error minimization

\section{Background}

With the development of recent decades, the performance of the cochlear implant has been greatly improved, and the users can have a very smooth face-to-face conversation and telephone communication in a quiet environment. However, in the noisy environment, the performance of the cochlear implant is significantly reduced, and the speech recognition rate is significantly reduced [1-5]. In order to improve the speech recognition ability of cochlear implant, the current research focuses on the fine structure coding, optical cochlear, electric field focusing, current 
guiding, virtual electrode, microphone array speech enhancement and so on. Among them, the microphone array speech enhancement method is based on the situation that the target speech signal and interference noise have different directions in the use scene [6-8]. In the practical application of cochlear implant, users want to enhance the forward signal in face-to-face communication, but they don't pay much attention to the signal from other directions. Research shows that for $50 \%$ sentence recognition rate, the signal to noise ratio (SNR) required by normal people is about $-10 d B$, while the SNR required by cochlear implant users is between 5 and $15 d B$; however, the SNR of daily living environment is usually 5 to $10 d B$ [9-10]. Therefore, under the noise of normal living environment, the speech recognition rate of cochlear implant users is difficult to reach more than $50 \%$. Because the speech recognition rate of the cochlear implant in the quiet environment has been relatively high, improving the SNR of its front-end signal acquisition is equivalent to making the cochlear implant work in the "quiet" environment, which is conducive to improving the performance of the cochlear implant. In recent years, microphone array technology has been widely used in video conference, car handsfree telephone, hearing aid and so on. Also, it has appeared in the research of front-end acquisition for cochlear implant, using microphone array speech enhancement technology to enhance the speech signal in the front-end device of cochlear implant. The microphone array method uses multiple sound sensors to collect sound signals, which helps to separate the target signal from the noise signal. This method can improve the SNR of the cochlear implant, and then improve the speech recognition rate [11-13].

The core of microphone array technology is delay and sum beamforming (DSB). In this method, multiple microphone sensors are placed in space to collect multichannel sound signals, and the corresponding channel signals are given certain delay and gain parameters. Finally, the output signals with directional characteristics are obtained to form different responses to signals in different directions. In essence, beamforming realizes spatial filtering, that is, the system response realizes the directional adjustment of signal amplitude, the enhancement of target orientation signal and the suppression of ambient signal. Because the recognition rate of cochlear implant in quiet environment is high, but it is low in noise environment, if the cochlear implant can be restored to work in "quiet" environment, it is expected to improve its performance. In the noisy environment, the signals collected by the front-end of the cochlear implant contain a lot of noise. If the SNR of signal acquisition can be improved, the interference noise will be weakened when it is transmitted to the speech processor of the cochlear implant, which is helpful to improve the speech recognition rate of the wearer. In addition, the common situation in the daily use of cochlear implant is the separation of target voice and interference noise in the spatial direction. What users want to solve most is that when they are in face-to-face communication, the voice or environmental noise and reverberation of the nearby speaker can be effectively removed. Therefore, the cochlear implant microphone array technology is helpful to improve speech recognition rate. 
In microphone array beamforming, the delay value is the main parameter to determine the beam shape. By changing the delay and other parameters, different directional beam modes can be designed [14-16]. For example, in reference [14], multiple beam patterns can be designed by using different coefficients in the mode of four acoustic tubes, and in reference [15], different beam patterns can be obtained by using two microphone modes with different delay and gain coefficients. In particular, different delay values have a great impact on the beam pattern of the dual microphone system [17-18]. Due to the size limitation of cochlear implant, the distance between microphones is very small. For a specific sampling rate, the delay value corresponds to the fractional sampling point [19-20]. Since the signals collected at the front-end of the cochlear implant have become discrete signals, it is necessary to realize the delay signal by interpolation on the basis of the original discrete signals [21]. The least mean square fractional delay filter [22-27] and the maximal flat fractional delay filter [23-32] are the most widely used fractional delay interpolation methods. Among them, the advantage of the least mean square fractional delay filter is to reduce the total mean square error of the whole frequency band, the disadvantage is that the local error is not minimum, and the global error is still large. The maximal flat fractional delay filter can minimize the error at low frequency or local position, but it has larger error at other frequencies and larger global error. Therefore, the previous methods do not solve the problem of global error minimization, and the error of global fractional delay in all frequency bands of the filterbank is still large. Because the filterbank of cochlear implant contains the process of frequency band division, the frequency range of each subband is relatively narrow. A fractional delay algorithm based on subband error minimization is proposed. The algorithm reduces the error of the center frequency of each subband to the minimum, and the error of each subband itself is very small, so that the fractional delay error of the whole speech band is minimized.

\section{Methods}

At present, the mainstream speech processing strategies for cochlear implant are based on filterbank, such as CIS, ACE and SPEAK. CIS strategy only transfers the parameters of one channel to the electrode at a time, and stimulates in order, and forms alternating electrical stimulation through continuous interleaved sampling. The stimulation rate range of CIS strategy is 740-2400 pulses per second. The SPEAK strategy and ACE strategy extract several frequency bands with largest energy information for electrode stimulation, and the extracted frequency bands are not equal interval or continuous interleaved stimulation. Because the energy of speech signal is mainly concentrated in the low-frequency band, the SPEAK strategy and ACE strategy are mainly to extract and transmit channel information in the low-frequency band. SPEAK strategy and ACE strategy are similar in algorithm architecture, and the specific parameters used are different. The stimulation rates of the two speech processing strategies are different, and ACE strategy has a higher stimulation rate. On the other hand, the number of channels of the two speech processing strategies is different. The SPEAK strategy sets 20 channels and selects the corresponding maximal 5-10 frequency bands for electrode stimulation, while the ACE strategy sets 22 channels 
and selects the channels within 20 for information transmission. Although the three mainstream strategies have different algorithm structures, they are all based on the filterbank mode. Therefore, the fractional delay filter algorithm studied in this paper is also based on the filterbank architecture.

Assuming that the signals collected from the front-end of the cochlear implant are expressed as $x(n)$. For a $M$-channel cochlear implant, subband signals $x_{i}(n)$ are separated from each channel, where $i$ ranges from 0 to $M$. The original signal $x(n)$ consists of subband signals, as shown in equation (1).

$$
x(n)=\sum_{i=1}^{M} x_{i}(n)
$$

In microphone array beamforming algorithm, $\tau$ is used to represent the value of delay parameter. For a sampling rate of $f_{s}$, the number of delay sampling points is $\tau f_{s}$. The delayed signal is shown in equation (2).

$$
x\left(n-\tau f_{s}\right)=\sum_{i=1}^{M} x_{i}\left(n-\tau f_{s}\right)
$$

For practical parameters, the delay sampling point $\tau f_{s}$ is not always an integer. It can be divided into an integer delay $D_{i}$ and a decimal delay $D_{d}$, as shown in equation (3).

$$
\tau_{s}=D_{i}+D_{d} \quad\left(0 \leq D_{d}<1\right)
$$

In equation (3), when $D_{d}$ is 0 , delay $\tau f_{s}$ is simplified to integer point delay. Its implementation is simple, only the shift of digital signal. When $D_{d}$ is not zero, delay $\tau_{s}$ is essentially interpolated between sampling points of known signal $x(n)$. For an ideal fractional delay system, the Ztransform is shown in equation (4).

$$
H_{\text {ideal }}(z)=z^{-\tau f_{s}}
$$

Equation (4) shows that the ideal fractional delay system is an all-pass system with linear phase, which is a non-causal system and cannot be implemented in real time, and cannot be directly used in real-time signal processing in cochlear implant. Considering the characteristics of signal processing and the stability of the algorithm in cochlear implant, the FIR fractional delay filter is designed to approximate the ideal filter and minimize the delay error. Because the speech processing strategy of the cochlear implant has been divided into frequency bands, each subband contains a corresponding narrow-band signal. The coefficients of the corresponding fractional delay filter are different because of the different frequency bands. For the subband of channel $i$, the unit impulse response $h_{i}(n)$ of the designed $K$-order fractional delay filter can be expressed as equation (5).

$$
h_{i}(n)=\beta_{0, i} \delta(n)+\beta_{1, i} \delta(n-1)+\beta_{2, i} \delta(n-2)+\cdots+\beta_{K, i} \delta(n-K)
$$

where $\beta_{0, i}, \beta_{1, i}, \ldots, \beta_{K, i}$ are the coefficients of $K$-order FIR fractional delay filters designed for the $i$ subband of the cochlear implant. The length of the filter is $K+1$, and the corresponding system frequency response function in Z-transform is $H_{i}(z)$. The error function $E_{i}(z)$ of the 
designed $K$-order FIR fractional delay filter and ideal fractional delay filter in $\mathrm{Z}$ domain is shown in equation (6).

$$
\begin{aligned}
& E_{i}(z) \\
& =H_{i}(z)-H_{\text {ideal }}(z) \\
& =\beta_{0, i}+\beta_{1, i} z^{-1}+\beta_{2, i} z^{-2}+\cdots+\beta_{K, i} z^{-K}-H_{\text {ideal }}(z)
\end{aligned}
$$

By substituting formula (4) into formula (6), the expression of error function in the $i$ subband is shown in formula (7).

$$
E_{i}(z)=\beta_{0, i}+\beta_{1, i} z^{-1}+\beta_{2, i} z^{-2}+\cdots+\beta_{K, i} z^{-K}-z^{-\tau f_{s}}
$$

It can be seen that the error function $E_{i}(z)$ has at least $K$-order derivatives, and the Taylor series can be used to expand the function $E_{i}(z)$. Since the error function $E_{i}(z)$ is based on the signal of the $i$ subband, the position of the central angular frequency $\omega_{c e n, i}$ of this frequency band can be selected to expand the error function $\left(\omega_{c e n, i}=2 \pi \cdot f_{c e n, i}\right.$, where $f_{c e n, i}$ is the central frequency of the $i$ subband). In Z-domain, $\omega_{c e n, i}$ corresponds to $z_{c e n, i}$. The Taylor series expansion form of the error function is shown in equation (8).

$$
\begin{aligned}
& E_{i}(z) \\
& =E_{i}\left(z_{c e n, i}\right)+\left.\frac{\mathrm{d} E_{i}(z)}{\mathrm{d} z}\right|_{z=z_{c e n, i}} \Delta z+\frac{\left.\frac{\mathrm{d}^{2} E_{i}(z)}{\mathrm{d} z^{2}}\right|_{z=z_{c e n, i}}}{2 !} \Delta z^{2}+\cdots \\
& +\frac{\left.\frac{\mathrm{d}^{K} E_{i}(z)}{\mathrm{d} z^{K}}\right|_{z=z_{c e n, i}}}{K !} \Delta z^{K}+o\left(\Delta z^{K}\right)
\end{aligned}
$$

where $\Delta z=z_{i}-z_{\text {cen,i }}$, and $o\left(\Delta z^{K}\right)$ is the higher-order infinitesimal quantity.

When $E_{i}(z)$ satisfies the following conditions of derivatives are 0 and error function value at the center frequency is also 0 (see equation (9)), then $E_{i}(z)=o\left(\Delta z^{K}\right)$, and the error can be minimized.

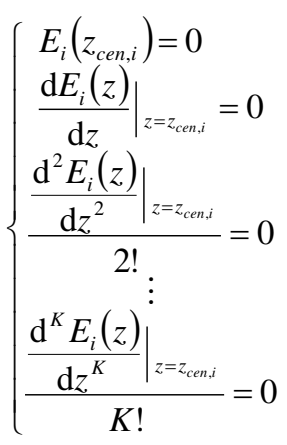

The following equations can be obtained by substituting equation (7) with equation (9), and change to the expression of corresponding angular frequency $\omega_{c e n, i}$. 


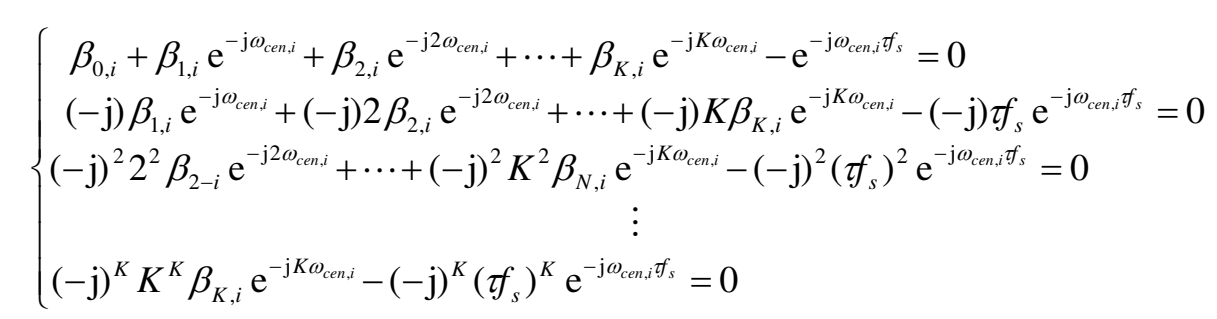

The coefficients of the unit impulse response of the designed filter can be obtained by using Cramer's rule [33-35] for the linear equations in equations (10). Corresponding coefficients of $\beta_{0, i}, \beta_{1, i}, \ldots, \beta_{K, i}$ are shown in equation (11).

$$
\left\{\begin{array}{c}
\beta_{0, i}=\frac{\left(\tau f_{s}-1\right)\left(\tau f_{s}-2\right) \cdots\left(\tau f_{s}-K\right)}{(-1)(-2) \cdots(-K)} \mathrm{e}^{-\mathrm{j} \omega_{c e n, i} f_{s}} \\
\beta_{1, i}=\frac{\tau f_{s}\left(\tau f_{s}-2\right) \cdots\left(\tau f_{s}-K\right)}{(1)(-1) \cdots(1-K)} \mathrm{e}^{\mathrm{j} \omega_{c e n, i}\left(1-f_{s}\right)} \\
\vdots \\
\beta_{p, i}=\frac{\prod_{m=0, m \neq p}^{K}\left(\tau f_{s}-m\right)}{(-1)^{K-p} p !(N-p) !} \mathrm{e}^{\mathrm{j} \omega_{c e n, i}\left(p-f_{s}\right)} \\
\beta_{K, i}=\frac{\tau f_{s}\left(\tau f_{s}-1\right) \cdots\left(\tau f_{s}-K+1\right)}{(K)(K-1) \cdots(1)} \mathrm{e}^{\mathrm{j} \omega_{c e n, i}\left(1-f_{s}\right)}
\end{array}\right.
$$

From the previous analysis and deduction, for the delay parameters needed in the practical application of the cochlear implant, the coefficients of the required $K$-order fractional delay filter can be calculated by substituting the delay value $\tau$ and the corresponding frequency band's central angle frequency $\omega_{c e n, i}$. The error is a high-order infinitesimal quantity $o\left(\Delta z^{K}\right)$. At the same time, after the frequency band division of the cochlear implant, each frequency band is narrow-band. The value of $\Delta z$ (corresponding to $\Delta \omega$ ) is small, so the fractional delay error of each subband is small.

\section{Realization and results}

Due to the size limitation of cochlear implant, the distance between microphones is very small, generally between $1 \mathrm{~cm}$ and $2 \mathrm{~cm}$. For the common delay parameters of a $d / c$ ( $d$ is the intermicrophone distance, $c$ is the sound speed), the delay parameters ranges from $29.41 \mu \mathrm{s}$ to $58.82 \mu \mathrm{s}$. And the number of commonly used cochlear implant channels is 16-24. There are many modes of frequency band division of filterbank in cochlear implant, such as FFT filterbank and Gammatone filterbank [36-37]. The algorithm proposed in this paper can be applied to different frequency band division modes, only through the cut-off frequency of each subband to calculate. Through this algorithm, the coefficients of fractional delay filters of different subbands can be calculated and embedded in the conventional continuous interleaved sampling strategy or the strategy of selecting the maximum amplitude (ACE, SPEAK, etc.), and the implementation flow is shown in figure 1 (take the system of two microphones as an example). 


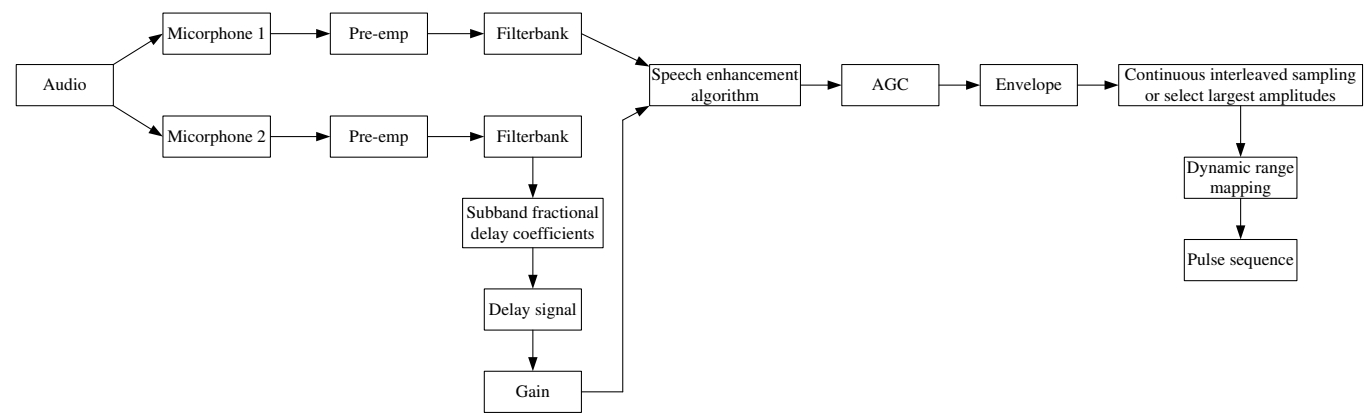

Fig. 1 Processing schemes of the subband fractional delay coefficients in the speech processing strategy of cochlear implant.

In figure 1, two microphones collect and form signals of two channels. The two signals are processed according to the conventional cochlear speech processing strategy, such as pre emphasis and frequency band division (FFT filterbank mode or Gammatone filterbank mode). Then one channel of signals is given the delay parameters of beamforming through the proposed subband fractional delay filter, and then the combination of gain parameters can be used for speech enhancement of microphone array algorithm. After embedding into the cochlear implant, the following signal processing processes are continued to be implemented, such as envelope extraction, channel selection, dynamic range mapping. Finally, the stimulus sequence was formed. Taking NRRO-TRON's 24-channel cochlear implant as an example, the filterbank are divided into 24 subbands: [152 274], [274 396], [396 517], [517 639], [639 761], [761 883], [883 1005], [1005 1127], [1127 1249], [1249 1432], [1432 1614], [1614 1797], [1797 2097 2041], [2041 2346], [2346 2650] [2650 2955], [2955 3321], [3321 3747], [3747 4235], [4235 4783], [4783 5392], [5392 6124], [6214 6916], [6916 7769] Hz, and the corresponding center frequencies are 213, 335, $456.5,578,700,822$, 944, 1066, 1188, 1340.5, 1523, 1705.5, 1919, 2193.5, 2498, 2802.5, 3138, $3534,3991,4509,5087.5,5758,6520,7342.5 \mathrm{~Hz}$. The fractional delay filter coefficients of corresponding subbands are calculated through the 24 selected center frequencies, and the amplitude frequency response of the third-order fractional delay filter with delay of $29.41 \mu \mathrm{s}$ is achieved at the sampling rate $f_{s}=22.05 \mathrm{kHz}$, as shown in figure 2 .

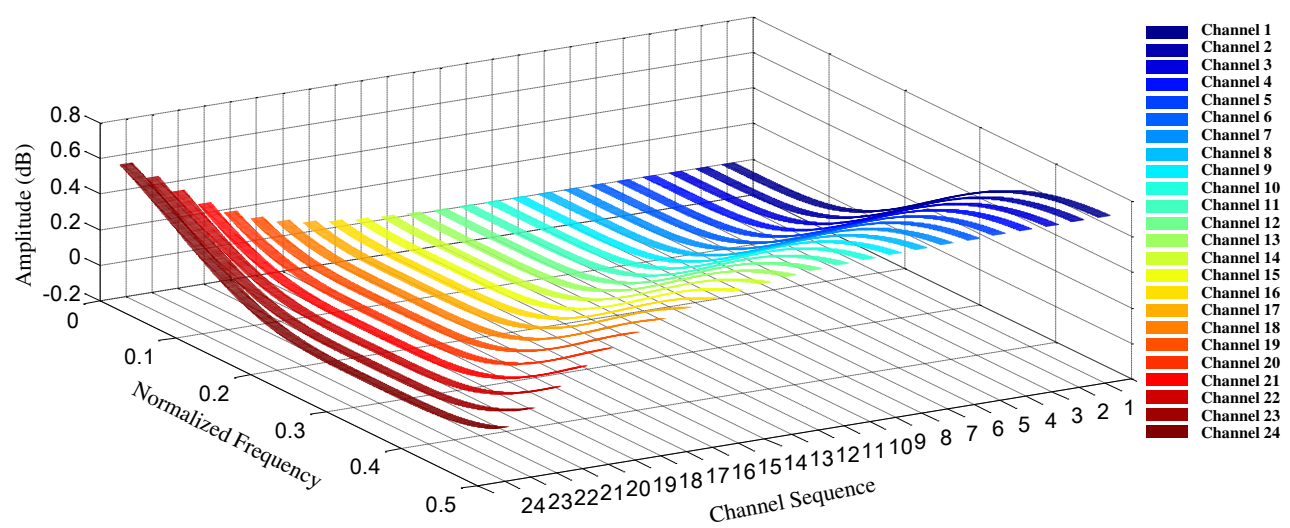

Fig. 2 Amplitude response for the proposed fractional delay filter in the 24-channel cochlear implant. 
Figure 2 shows the amplitude frequency response curve of the fractional delay filter in $d B$ at a sampling rate of $22.05 \mathrm{kHz}$, which corresponds to each frequency band of the 24 channel cochlear implant, with a delay of $29.41 \mu \mathrm{s}$ (based on the $\mathrm{d} / \mathrm{c}$ delay parameter and $1 \mathrm{~cm}$ inter-microphone distance). The subband channel numbers in the figure range from 1 to 24 . The center frequency of channel 1 is $213 \mathrm{~Hz}$ and that of channel 24 is $7342.5 \mathrm{~Hz}$. The ideal fractional delay filter is an all pass system with linear phase, and its amplitude frequency response is 1 (corresponding to $0 d B$ ). Therefore, the closer the amplitude frequency response of the designed fractional delay filter is, the higher its accuracy is. From the shape of the amplitude frequency response curve of the 24 channel subband fractional delay filter in the figure, it can be seen that when the frequency is low, the amplitude frequency response curve is close to the low frequency position, while when the frequency is gradually increased, the approximate position of the amplitude frequency response curve changes from the low frequency to the intermediate frequency, and then to the high frequency position. Because each subband in the filterbank contains only narrowband signal, the designed fractional delay filter achieves the characteristic that the amplitude frequency response curve is close to the ideal fractional delay filter in the corresponding subband. The deviation of the amplitude frequency response value of the system at other positions of the filter does not affect the accuracy of the fractional delay in the corresponding subband, because the designed subband fractional delay filter only uses the response curve of the system in this frequency band, and uses other curves outside the frequency band.

Since each subband in the filterbank of cochlear implant is a narrow-band signal, the effective part of the amplitude frequency response curve of the fractional delay filter system is limited to that part of the curve in the corresponding subband. The effective subband response curves of each frequency band of 24-channel cochlear implant can form a full-band amplitude frequency response curve, as shown in Fig. 3.

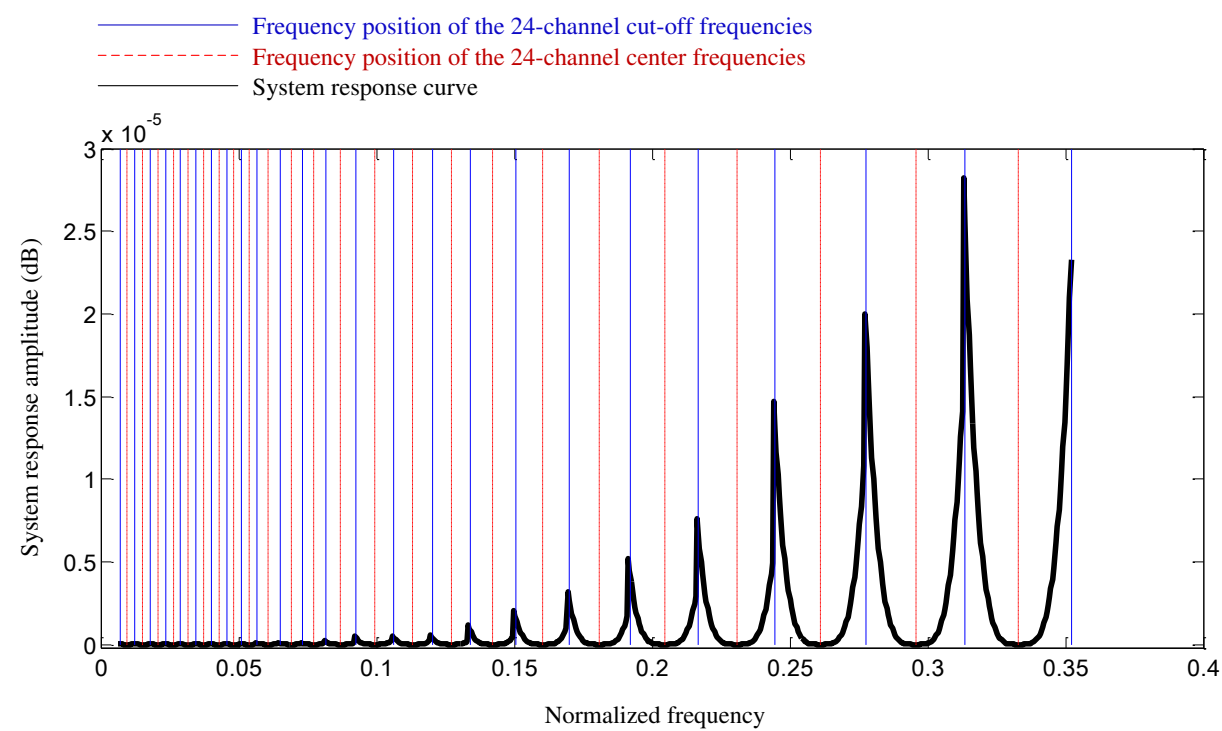

Fig. 3 Response curve of 24-channel full-band fractional delay filter system. 
Figure 3 shows the system response curve of 24-channel full-band fractional delay filter for cochlear implant. For an ideal fractional delay filter, the amplitude frequency response of the system is constant to $1(0 d B)$. The closer the amplitude frequency response of the designed fractional delay filter is to 1, the higher the accuracy is. As can be seen from figure 3, the maximum deviation of the system response value of the designed fractional delay filter in the whole frequency band of the 24-channel filterbank is $3 \times 10^{-5} d B$. As $3 \times 10^{-5} d B$ corresponds to 1.000007 , that is to say, the maximum deviation is $0.0007 \%$. Therefore, the error of the fractional delay filter designed in this paper is extremely small, which can meet the requirements of cochlear implant using microphone array technology for the accuracy of delay parameters.

\section{Discussions}

In the application of front-end microphone array for cochlear implant, the range of delay parameters is $0 \sim d / c$. For different delay values, the error of fractional delay filter is different. Similarly, the error of filterbank with different number of channels of cochlear implant is also different. The distance between microphones used at the front-end of the cochlear implant is very small. The value of $d$ is usually $1 \sim 2 \mathrm{~cm}$, so the value range of $d / c$ is $29.41 \mu \mathrm{s} \sim 58.82 \mu \mathrm{s}$. When sampling rate is $22.05 \mathrm{kHz}$, the maximum delay point of $d / c$ is 1.297 , and when sampling rate is $44.1 \mathrm{kHz}$, the maximum delay point of $d / c$ is 2.594 . For integer delay points, the maximum number of delay points is no more than 3 in the application of cochlear implant. Therefore, the errors of fractional delay filters of different filters can be analyzed when the sampling points are delayed from 0 to 3 . Although the range is only $0 \sim 3$, the value can be continuous, and any small value can be taken in the range. If the error of the designed fractional delay filter is small and the delay interval is small enough, the resolution is high enough.

Because the errors of different delaying values are different, the average errors can be calculated, as shown in figure 4.

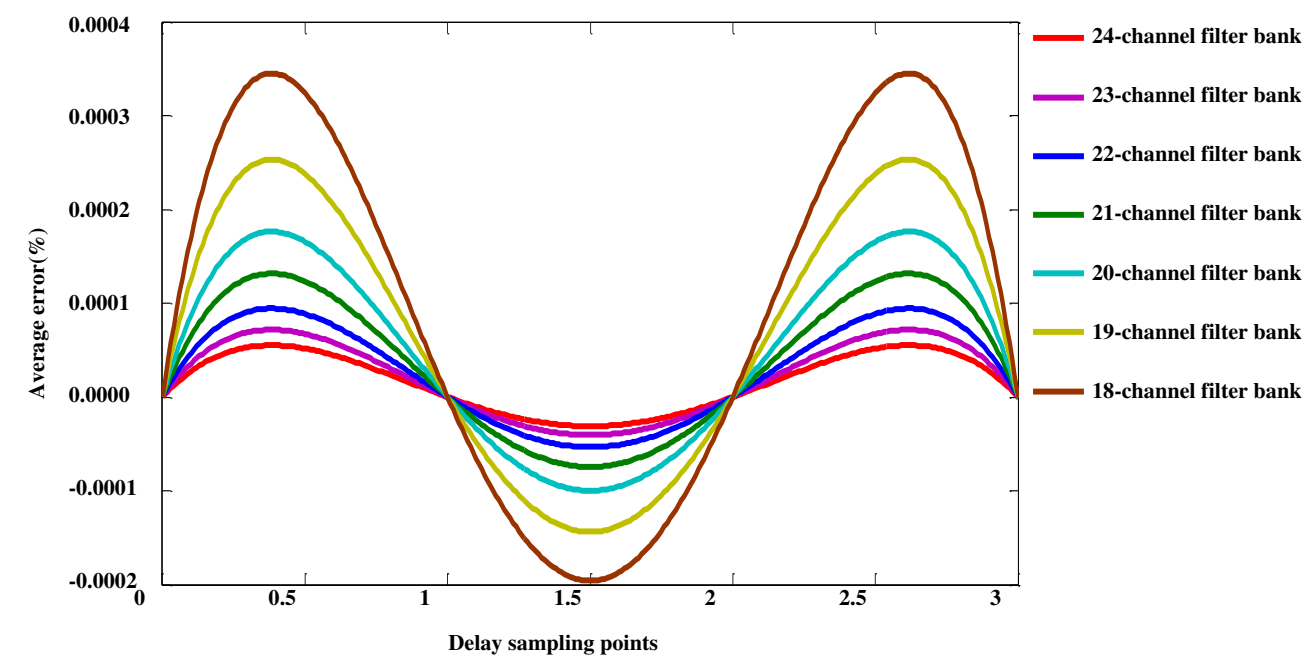

Fig. 4 Average error of fractional delay filters with different delay and channel quantity. 
Figure 4 shows the average error of fractional delay filters with different delay and channel quantity. It can be seen from the figure that there are two cases of average error: positive error and negative error. In different cases, the average error is very small, not more than $0.0004 \%$. When the number of channels is large, the average error is smaller, and when the number of channels is small, the average error tends to increase. In figure 4, the delay values range from 0 to 3 , where 0 , 1,2 , and 3 are sampling points for delay integers and others are sampling points for delay decimals. It can be seen that the error of delaying integer sampling points is 0 , so the average error is gradually increasing and then decreasing. Therefore, the maximum error occurs between two integer sampling points. The calculation shows that the position of delay parameter for the maximum error in the range of $0 \sim 1$ is 0.385 , and the 16 24 channel filterbank all have the same results. Similarly, the position of delay parameter for the maximum error in the range of $1 \sim 2$ is 1.5 , and the position of delay parameter for the maximum error in the range of $2 \sim 3$ is 2.615 . The filterbank with different channel numbers have similar characteristics, and the average error is very small, which is helpful to realize the precise delay value in the algorithm of front-end microphone array beamforming.

\section{Conclusion}

In microphone array technology, delay value is an important parameter of beam design, whose accuracy determines the stability of beam pattern. Due to the limitation of cochlear implant size, the distance between microphones is very small, and the delay value is also very small, which leads to the fractional delay problem in the application field of cochlear implant. In this paper, a subband fractional delay method is proposed based on the frequency band division characteristics of filterbank in cochlear implant. The fractional delay filter designed by this method can minimize the local and global fractional delay errors, and solve the problem that the whole frequency band fractional delay error is too large in the previous technology. The algorithm can be embedded into the speech processing structure of cochlear implant, which has important theoretical and engineering value.

\section{Acknowledgments}

This work was support by natural science foundation of Guangdong province under the grant No. 2018A030313146, by the characteristic innovation projects of Guangdong Universities in 2019 under the grant No. 2019GKTSCX094, by national natural science foundation of China under the grant No. 61704113.

\section{Author details}

${ }^{1}$ Room 1603B, Science and Technology Building, Shenzhen Institute of Information Technology, Shenzhen, P.R. China, 518000.

${ }^{2}$ Room 1603D, Science and Technology Building, Shenzhen Institute of Information Technology, Shenzhen, P.R. China, 518000.

\section{Authors' contributions}

Yousheng Chen initiated and conceived the algorithm, designed simulation experiments and analyzed the algorithm feature. Yan Chen is the corresponding author, she is responsible for revising this manuscript. All authors read and approved the final manuscript. 


\section{Competing interests}

The authors declare that they have no competing interests.

\section{Ethics approval and consent to participate}

Not applicable.

\section{Consent for publication}

The main content of this paper is theoretical analysis and simulation, which is publishable.

\section{Availability of data and material}

The program code used in this study are available from the corresponding author on reasonable request.

\section{Funding}

This research was funded by natural science foundation of Guangdong province under the grant No. 2018A030313146, by the characteristic innovation projects of Guangdong Universities in 2019 under the grant No. 2019GKTSCX094, by national natural science foundation of China under the grant No. 61704113.

\section{References}

1. F.G. Zeng. Challenges in Improving Cochlear Implant Performance and Accessibility. IEEE Transactions on Biomedical Engineering, vol.64, no.8, pp.1662-1664, 2017.

2. Y.S. Chen, J. Wang, G.W. Xue, et al. Research on parameter feature of continuous interleaved sampling strategy for cochlear implant. Journal of Shenzhen Institute of Information Technology, vol.15, no.3, pp.12-18, 2017.

3. Y.S. Chen, W.F. Chen. Research on intelligent terminal-based medical surveilliance system for cochlear implant. Beijing Biomedical Engineering, vol.36, no.4, pp.400-407, 2017.

4. Y.S. Chen, J. Wang, G.W. Xue, et al. Vibration and mismatch of near-field signal acquisition for cochlear implant. Chinese Journal of Medical Physics, vol.34, no.7, pp.731-735, 2017.

5. F.G. Zeng. Trends in cochlear implant. Trends Amplif, vol.8, no.1, pp. 1-34, 2004.

6. T. Ogawa, S. Takada, K. Akagiri, et al. Speech enhancement using a square microphone array in the presence of directional and diffuse Noise. IEICE T FUND ELECTR, vol.93, no.5, pp.926-935, 2017.

7. T. Kawase, K. Niwa, M. Fujimoto, et al. Integration of Spatial Cue-Based Noise Reduction and Speech Model-Based Source Restoration for Real Time Speech Enhancement [J]. IEICE T FUND ELECTR, vol.100, no.5, pp.1127-1136, 2017.

8. I. Mosnier, N. Mathias, J. Flament, et al. Benefit of the UltraZoom beamforming technology in noise in cochlear implant users. European Archives of Oto-Rhino-Laryngology, vol.274, no.9, pp.3335-3342, 2017.

9. P.B. Nelson, S.B. Jin, A.E. Carney. Understanding speech in modulated interference: cochlear implant users and normal-hearing listeners [J]. J Acoust Soc Amer, 2003, 113(2): 961-968.

10. F.G. Zeng, K. Nie, G.S. Stickney, et al. Speech recognition with amplitude and frequency modulations [J]. Proc Nat Acad Sci USA, 2005, 102(7): 2293-2298.

11. Y.S. Chen, Q. Gong. Real-time spectrum estimation-based dual-channel speech-enhancement algorithm for cochlear implant. Biomed Eng Online, vol.11, no.74, 2010.

12. M. Ryoji, S. Akihiko. A directional noise suppressor with a specified constant beamwidth. IEICE Transactions on Fundamentals of Electronics, Communications and Computer Sciences, vol.101, no.10, pp.1616-1624, 2018.

13. M. Ryoji, S. Akihiko. Gain relaxation: a solution to overlooked performance degradation in speech recognition with signal enhancement. IEICE Transactions on Fundamentals of Electronics, Communications and Computer Sciences, vol.101, no.11, pp.1832-1840, 2018.

14. Y.S. Chen, Y. Chen. Research of front-end speech enhancement and beamforming algorithm based on dual microphone for cochlear implant. Journal of Biomedical Engineering, vol.36, no.3, pp.1-10, 2019.

15. Y.S. Chen, C.X. Zhang, W.Z. Lin, et al. Beam pattern characteristics of directional microphone and features of gain changes in cochlear implant. Chinese Journal of Medical Physics, vol.35, no.12, pp.1468-1472, 2018.

16. Y. Hioka, K. Kobayashi, K. Furuya, et al. Enhancement of sound sources located within a particular area using a pair of small microphone arrays. IEICE Transactions on Fundamentals of Electronics, Communications and Computer Sciences, vol.91, no.2, pp.561-574, 2008.

17. Y.S. Chen, C.X. Zhang, W.F. Chen et al. Dual-microphone based signal acquisition and different parameters based beamforming features for cochlear implant. Chinese Journal of Medical Physics, vol.35, no.7, pp.811-815, 2018.

18. Q. Gong, Y.S. Chen. Parameter selection methods of delay and beamforming for cochlear implant speech enhancement. Acoustical Physics, vol.57, no.4, pp.542-550, 2011. 
19. C.C. Tseng. Improved design of digital fractional-order differentiators using fractional sample delay. IEEE Transactions on Circuit and Systems, vol.53, no.1, pp.193-203, 2006.

20. S. Paquelet, A. Zeineddine, A. Nafkha, et al. Convergence of the Newton structure transfer function to the ideal fractional delay filter. IEEE Signal Processing Letters, vol.26, no.9, pp.1354-1358, 2019.

21. Y.S. Chen, Q. Gong. Small-space microphone array fractional delay algorithm based on FIR filter for cochlear implant. Tsinghua Sci Technol, vol.16, pp.90-94, 2011.

22. Y.S. Chen, W.F. Chen. Research on fractional delay filter and mismatch feature based on least mean square rule for CI device. IHMSC 2017, Hangzhou, China, no.1, pp.308-311, 2017.

23. N.J. Sang, X.G. Wang, Y. Zhou. Adaptive cancellation in transmitted signal of repeating type jammer based on fractional delay filter. Proceedings of 2011 IEEE CIE international conference on radar, Chengdu, China, pp.1071$1074,2011$.

24. H. Zhao, J.B. Yu. A simple and efficient design of variable fractional delay FIR filters. IEEE Transactions on Circuits and Systems, vol.53, no.2, pp.157-160, 2006.

25. H.K. Kwan, A.M. Jiang. FIR, allpass, and IIR variable fractional delay digital filter design. IEEE Transactions on Circuits and Systems, vol.56, no.9, pp.2064-2074, 2009.

26. H.H. Dam. Design of allpass variable fractional delay filter. IEEE Transactions on Signal Processing, vol.59, no.12, pp.6240-6244, 2011.

27. W.R. Lee, L. Caccetta, V. Rehbock. Optimal design of all-pass variable fractional-delay digital filters. IEEE Transactions on Circuits and Systems, vol.55, no.5, pp.1248-1256, 2008.

28. J.P. Thiran. Recursive digital filters with maximally flat group delay. IEEE T Circuits-I, vol.18, no.6, pp. 659-664, 1971.

29. E. Hermanowicz. Explicit formulas for weighting coefficients of maximally flat tunable FIR delayers. Electron Lett, vol.28, no.20, pp.1936-1937, 1992.

30. S.C. Pei, P.H. Wang, H.S. Lin. Closed-form design of maximally flat FIR fractional delay filters. IEEE Signal Processing Letters, vol.13, no.7, pp.405-408, 2006.

31. S. Koshita, M. Abe, M. Kawamata. A simple ladder realization of maximally flat allpass fractional delay filters. IEEE Transactions on Circuit and Systems, vol.61, no.3, pp.203-207, 2014.

32. T.B. Deng. Symmetric structure for odd-order maximally flat and weighted-least-squares variable fractional-delay filters. IEEE Transactions on Circuit and Systems, vol.54, no.12, pp.2718-2732, 2007.

33. V. Valimaki, M. Karjalainen, U.K. Laine. Splitting the unit delay. IEEE Signal Process Mag, vol.13, no.1, pp. 30-60, 1996.

34. R.E. Klein. Teaching linear systems theory using Cramer's rule. IEEE Transactions on Education, vol.33, no.3, pp. 258-267, 1990.

35. M.C.M. Teixeira, H.F. Marchesi, E. Assuncal. Signal-flow graphs: direct method of reduction and MATLAB implementation. IEEE Transactions on Education, vol.44, no.2, pp. 185-190, 2001.

36. J. Laneau, J. Wouters, M. Moonen. Relative contributions of temporal and place pitch cues to fundamental frequency discrimination in cochlear implantees. Journal of the Acoustical Society of America, vol.116, pp. 3606-3619, 2004.

37. S. Tabibi, A. Kegel, W.K. Lai, et al. Investigating the use of a gammatone filterbank for a cochlear implant coding strategy. Journal of Neuroscience Methods, pp. 63-74, 2017. 


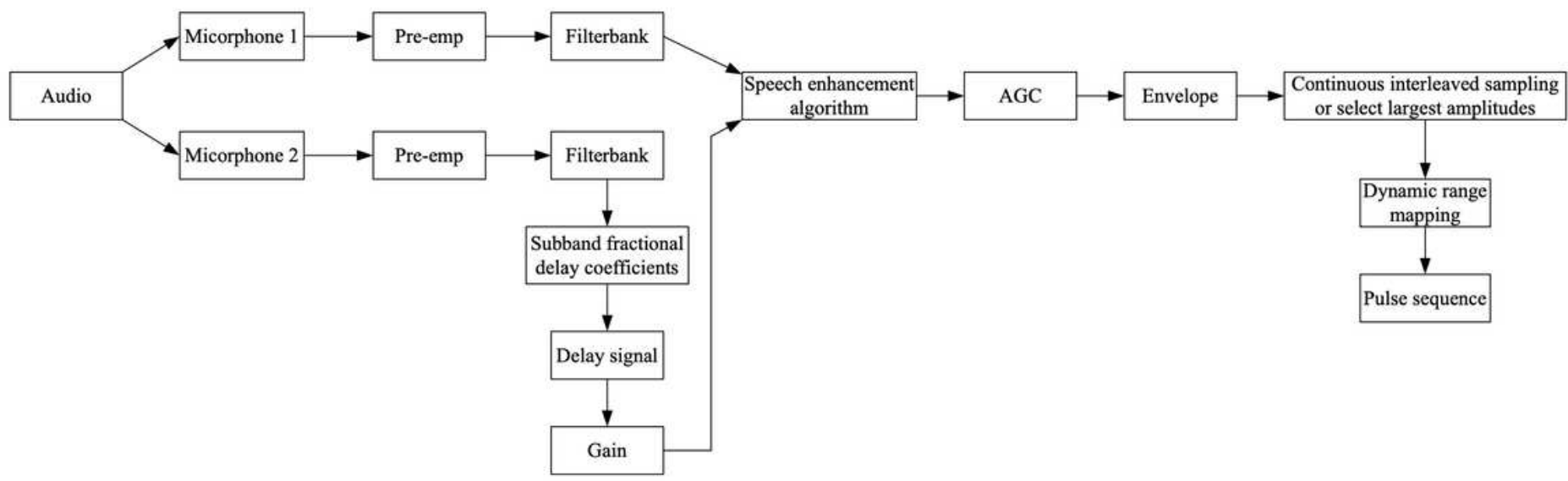

\section{Figure 1}

Processing schemes of the subband fractional delay coefficients in the speech processing strategy of cochlear implant.

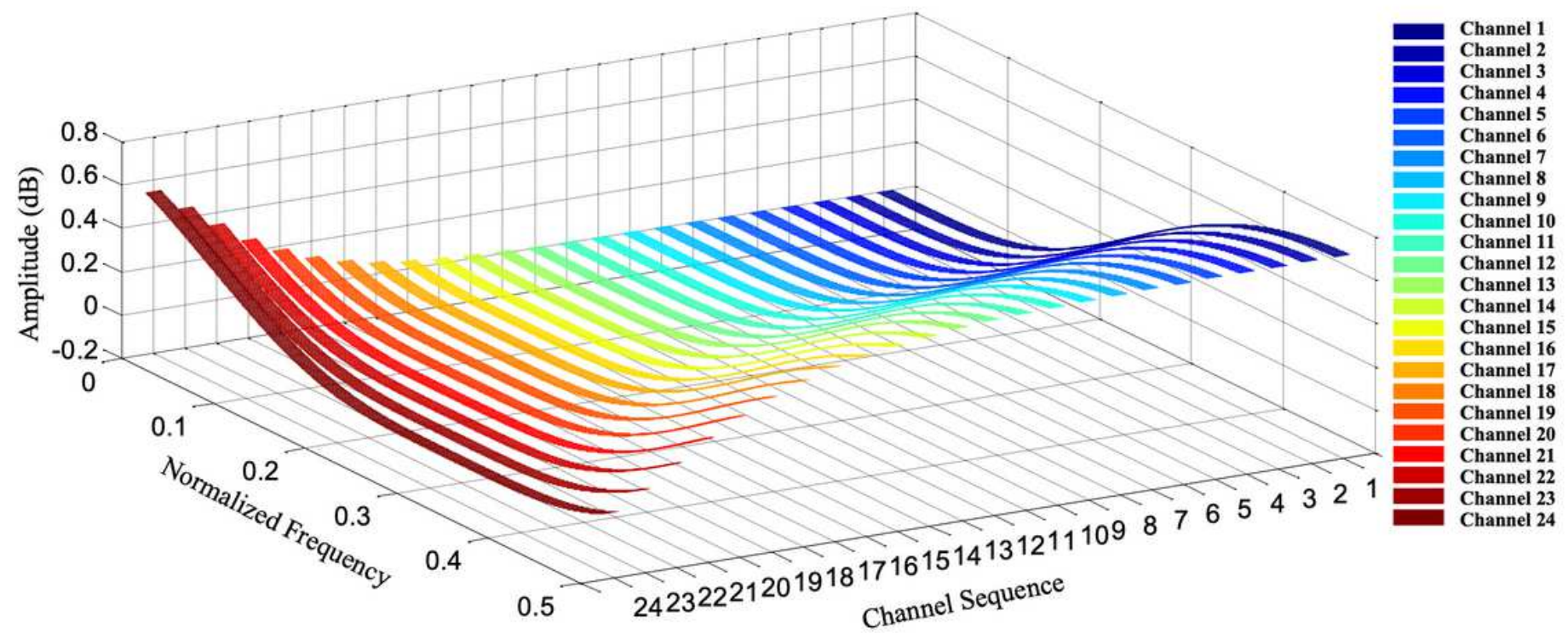

Figure 2

Amplitude response for the proposed fractional delay filter in the 24-channel cochlear implant. 
Frequency position of the 24-channel cut-off frequencies

Frequency position of the 24-channel center frequencies

System response curve

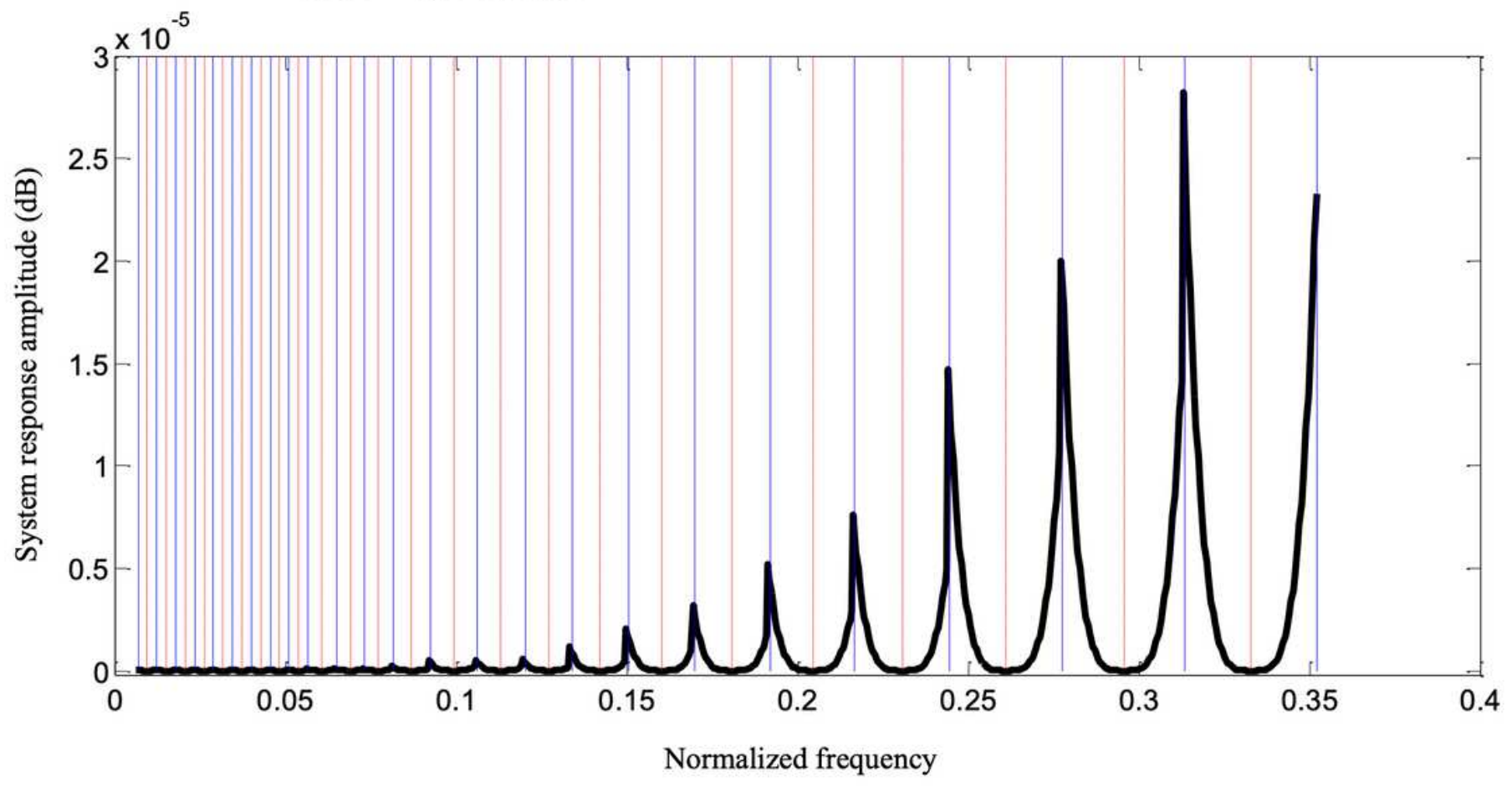

Figure 3

Response curve of 24-channel full-band fractional delay filter system.

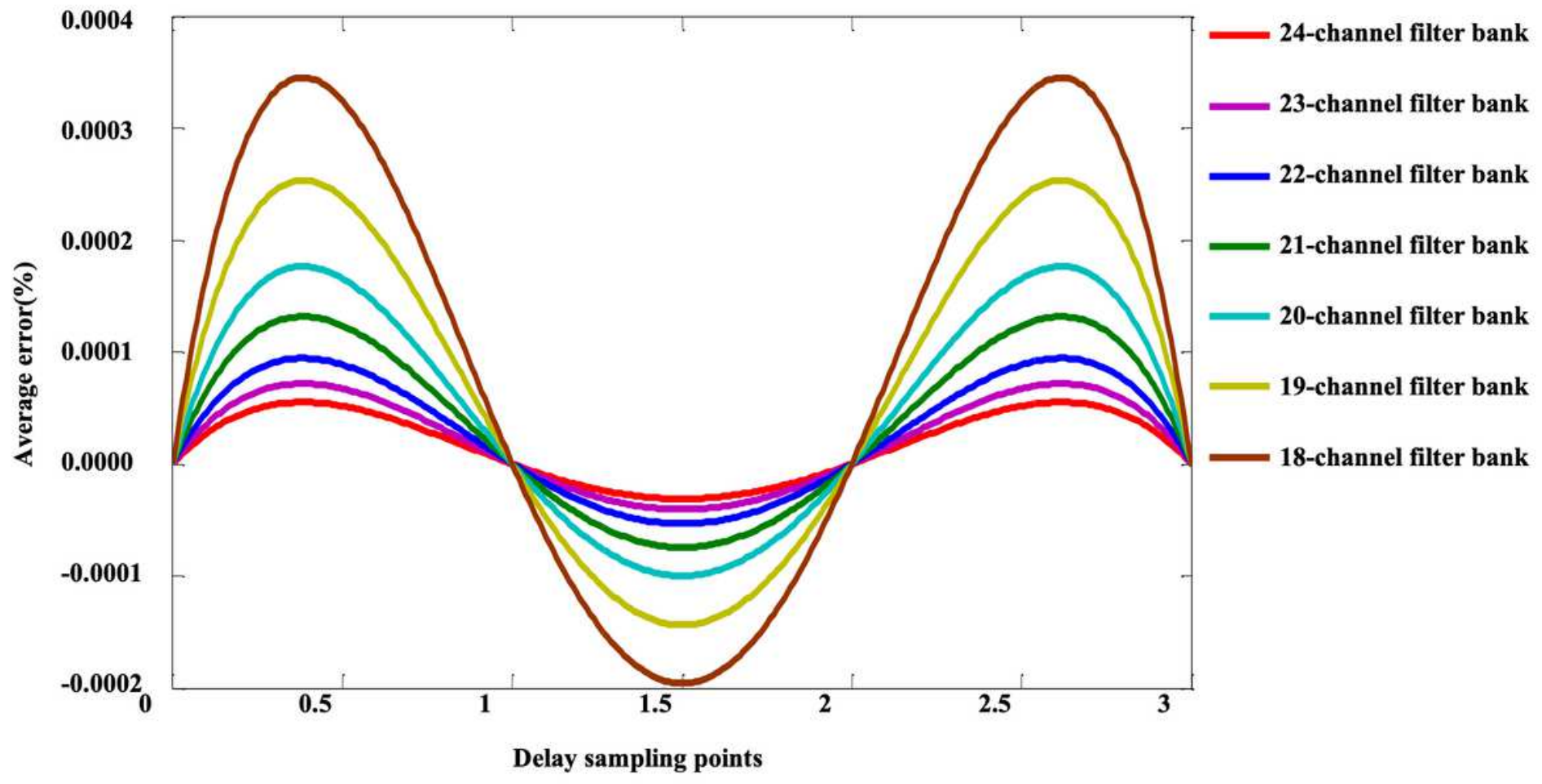

Figure 4 
Average error of fractional delay filters with different delay and channel quantity. 